Kozioł Magdalena, Lewicki Marcin, Pawlicki Mateusz, Lopuszyńska Anna, Krasa Aleksandra, Piekarska Ewa, Krasowska Danuta. A rare case of Raoultella planticola urinary tract infection. A case report with literature review. Journal of Education, Health and Sport. 2020;10(8):470- 475. eISSN 2391-8306. DOI http://dx.doi.org/10.12775/JEHS.2020.10.08.057

https://apcz.umk.pl/czasopisma/index.php/JEHS/article/view/JEHS.2020.10.08.057

https://zenodo.org/record/4009554

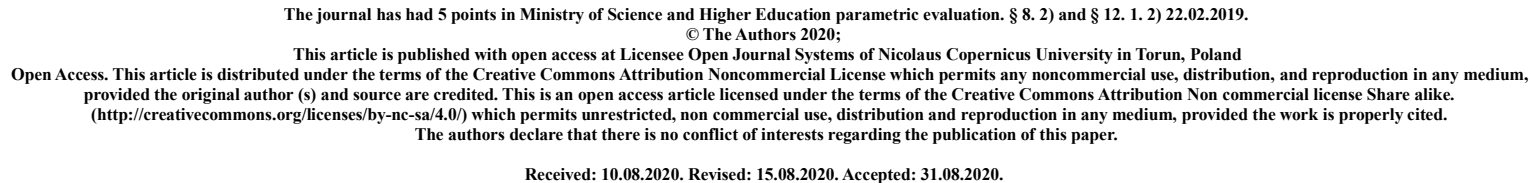

\title{
A rare case of Raoultella planticola urinary tract infection. A case report with literature review
}

\author{
Magdalena Kozioł ${ }^{a}$, Marcin Lewicki ${ }^{b}$, Mateusz Pawlicki ${ }^{a}$, Anna Lopuszyńskaa \\ Aleksandra Krasa ${ }^{a}$, Ewa Piekarska ${ }^{a}$, Danuta Krasowska ${ }^{c}$
}

astudent Scientific Association at Department of Epidemiology and Clinical Research Methodology, Medical University of Lublin, ul. Radziwiłłowska 11, Lublin 20-080, Poland; ${ }^{b}$ Department of Epidemiology and Clinical Research Methodology of the Medical University of Lublin, ul. Radziwiłłowska 11, Lublin 20-080, Poland;

'Students' Scientific Association at the Department of Dermatology, Venerology and Pediatric Dermatology, Medical University of Lublin, ul. Staszica 11, Lublin 20-081, Poland.

Corresponding author: Magdalena Kozioł, magdalena.koziol@icloud.com

\section{ORCID ID:}

Magdalena Kozioł https://orcid.org/0000-0002-8671-5968, magdalena.koziol@icloud.com Marcin Lewicki https://orcid.org/0000-0003-1906-9326, lewicki-marcin@wp.pl

Aleksandra Krasa https://orcid.org/0000-0002-0733-202X,ola.AK62@gmail.com Danuta Krasowska https://orcid.org/0000-0002-3015-1120, dana.krasowska@gmail.com Mateusz Pawlicki https://orcid.org/0000-0001-8318-6573, pawlak32@gmail.com Anna Łopuszyńska https://orcid.org/0000-0001-5133-4180, lopuszynskaania@gmail.com Ewa Piekarska https://orcid.org/0000-0002-4954-379X; piekarskaewaa@gmail.com 


\section{Abstract:}

Urine infections represent $40-50 \%$ of all infections in hospitalized patients. The most common uropathogens are E.coli, S.saprophitycus, and less commonly Enterobacteriaceae, such as Proteus mirabilis and Klebsiella pneumoniae. The spectrum of microorganisms isolated in hospital conditions may be wider. Raoultella planticola is a rare cause of human infections, so far only few cases of ZUM with this etiology have been described.

A 56-years-old patient, with a history of cardiac disease, with exacerbation of chronic kidney disease, was admitted to the Department of Internal Medicine. The reason for admission was general deterioration. Many abnormalities in laboratory tests were detected. The doctors' attention was focused on the significant bacteriuria. Moreover, the patient reported dysuria symptoms. Material for urine microbiological culture was taken and empirical antibiotic therapy with ciprofloxacin started. The obtained result of urine culture allowed to identify the bacteria by R. planticola. As a result of the applied treatment, the patient's condition improved.

An increasing number of infections with R. planticolla etiology is observed. The bacterium may lead to infections in many systems. The group of patients at risk of the disease includes people with renal dysfunction, who are immunosuppressed. Infections of R. planticolla etiology are usually characterized by good antibiotic response and positive prognosis.

\section{Key words: Raoultella planticola; urine infections; resistance}

\section{Introduction:}

Raoultella planticola from the family Enterobacteriaceae is a Gram negative, oxidasenegative, aerobic, poorly mobile bacteria without an envelope. It was first described in the 1980s as Klebsiella planticola and Klebsiella trevisanii [1], however, in 2001 it was reclassified to a new genus as Raoultella planticola together with Raoultella ornithinolytica and Raoultella terrigena [2]. This bacterium is commonly found in soil, water, aquatic plants, seafood [8] and clinically isolated from wounds, sputum, faeces and urine. A rare diagnosis of R. planticola infection may result from difficulties in the isolation and identification of this bacterium species - it is often confused with other types, e.g. Klebsiella spp. Infections are observed in immunosuppressed people, in the course of cancer [3-9,15], diabetes [10], in dialysis patients [11], using PPI. This pathogen attacks many systems without organic specification. The most common are urinary tract infections $[9,12,13,15]$, pneumonia $[3,4,14]$ and biliary tract infections $[5,6,7]$. In the case described by us, a patient with cachexia features was diagnosed with R. planticola's etiology of urinary tract inflammation.

\section{Case report:}

A 56-year-old patient with a history of cardiovascular disease (ischaemic heart disease, condition after double myocardial infarction, hypertension), intensification of chronic kidney disease, alcohol use disorder, hyperbilirubinemia and features of cachexia was admitted to the Department of Internal Medicine in emergency due to deterioration of general condition and episode of fainting. Hypotension and bradycardia was examined. 
Laboratory tests showed: hyperkalaemia $(\mathrm{K}+: 6.8 \mathrm{mmol} / \mathrm{l})$, increase in creatinine concentration to $3.13 \mathrm{mg} / \mathrm{dl}$ with eGFR decrease to $66.07 \mathrm{ml} / \mathrm{min}$, significantly increased urea level $(136.6 \mathrm{mg} / \mathrm{dl})$ and inflammatory parameters (CRP: $137.08 \mathrm{mg} / \mathrm{dl})$. NT-pro-BNP was $7153.0 \mathrm{pg} / \mathrm{ml}$, which confirmed the ischaemic heart disease. In the general urine test, significant bacteriuria was observed. The patient reported dysuric symptoms. Material for microbiological urine culture was collected and empirical antibiotic therapy with ciprofloxacin started. Adequate fluid therapy and pharmacological treatment was implemented. An examination for alarm pathogens was carried out. Swab for CPE: negative. A urine culture result was obtained which confirmed significant bacteriuria $(105 \mathrm{CFU} / \mathrm{ml})$ and identified the pathogen as R. planticola. The antibiogram showed resistance to ampicillin (MIC 16) with preserved sensitivity to inhibited beta-lactams, cephalosporins, amikacin, trimetroprim with sulfamethoxazole and fluoroquinolones. The current antibiotic therapy with ciprofloxacin has been maintained. As a result of the applied treatment the patient's condition was improved, haemodynamic parameters were stabilized, inflammatory markers were lowered (4-fold decrease of CRP concentration) and kidney function parameters were partially normalized with creatinine concentration reduction to $1.4 \mathrm{mg} / \mathrm{dl}$.

\section{Discussion:}

Hassan Mehmood et al. in 2018. described a similar case of a patient with diagnosed urinary tract infection of R. planticola etiology. A 65-year-old man with metabolic disease and end-stage renal failure as a result of $\operatorname{IgA}$ nephropathy, complained about dysuric symptoms and dark urine colour. In vital signs: blood pressure 164/89 $\mathrm{mm} \mathrm{Hg}$, heart rate 99/min, respiratory rate $20 / \mathrm{min}$, fever $39.3^{\circ} \mathrm{C}$. Physical examination shows subabdominal tenderness. Among the deviations in laboratory tests: neutrocytosis at normal WBC and creatinine $4.5 \mathrm{mg} / \mathrm{dL}$. Urine analysis showed the presence of bacteria, WBC $>50 / \mathrm{HPF}$. Ceftriaxone was administered intravenously. Urine culture showed the presence of $\mathrm{R}$. planticola bacteria, which were sensitive to all major antibiotics, including ceftriaxone, ciprofloxacin, nitrofurantoin, cefazolin, gentamicin and trimetoprim-sulfamethoxazole. As a result of antibiotic therapy, the patient was cured without complications [16].

Raoultella planticola has no specificity about the tissue involved; therefore, it may attack other organs and systems. Serkan Atıc1 et al. described the case of a 28-week-old infant weighing 880 grams, born prematurely due to preeclampsia. On the 34th day of life, bilateral purulent eye discharge was detected. Netylmycin was used empirically. The swab taken showed the presence of R. planticola. The antibiogram revealed resistance of the strain to ampicillin and piperacilin and sensitivity to cefuroxime and netylmycin, among others. The administration of the selected antibiotic was continued, however, despite the bacterial sensitivity to the administered antibiotic, the patient presented clinical symptoms of sepsis after several days, which induced the change of antibiotic to meropene and vancomycin. As a result of the applied treatment, the patient's health condition improved. The literature also describes cases of biliary tract occupation [5,6,7], lungs [3,4,14], pancreas [21], peritoneum [11], prostate [20], pleura, soft tissues after surgery [17].

The risk factors of R. planticola infection include kidney damage, cachexia, posttransplant condition, and immunosuppression. 2016r. 
William Paul Skelton and others described the case of a 73-year-old woman with stage IIIA multiple myeloma. The patient underwent treatment with four cycles of cyclophosphamide bortezomib-dexamethasone, followed by autologous stem cell transplantation after conditioning with melfalan. Three years later, as a result of a relapse, the patient was again treated with chemotherapy and a new peripheral blood stem cell transplantation was performed. The patient complained about loose stools, which were treated orally with vancomycin (etiology of Clostridium difficile). The study showed elevated body temperature to $39.3 \mathrm{C}$, hypotension $94 / 66 \mathrm{~mm} \mathrm{Hg}$, tachycardia 108/min and leukocytosis about 10.1 thousand/mm3. The presence of respiratory viruses and Giardia, Cryptosporidium, Vibrio, Yersinia and parasite eggs were excluded. After a week, the ongoing fever was accompanied by dysuric symptoms. The urine analysis showed the presence of nitrites and $230 \mathrm{WBC} / \mathrm{ww}$. Empirically cephalexin was applied, waiting for the culture result. Raoultella planticola cultured showed sensitivity to amikacin, cefoxitin, ciprofloxacin, levofloxacin, meropene, nitrofurantoin, piperacillin/tazobactam and trimetoprim/sulfamethoxazole. These bacteria were resistant to ampicillin, cefazolin, ceftazidim, ceftriaxon, gentamicin and triamycin. The treatment was continued for 7 days with $100 \mathrm{mg}$ of nitrofurantoin twice a day [9]. The immunosuppression induced by chemotherapy, steroids and cachexia probably explains why the above-mentioned patient was predisposed to Raoultella planticola infection.

The sensitivity to R. planticola antibiotics has not yet been precisely tested. Many studies have demonstrated that R. planticola species are usually sensitive to third or fourth generation cephalosporins, $\beta$-lactam antibiotics with $\beta$-lactamase inhibitors, aminoglycosides, netylmycin, ciprofloxacin, levofloxacin, tigecycline and carbapenems [16]. However, R. planticola may acquire resistance to antibiotics by plasmid. The possibility of R. planticola resistance for carbapenems should be kept in mind. Tseng SP et al. and $\mathrm{Xu} \mathrm{Mu}$ et al. separately described the cases of two pneumonia patients and isolated a strain of R. planticola bacteria resistant to carbapenems[3,18]. Jose Armando Gonzales Zamora et al. in 2018. described a case of a patient with second-degree burns and pneumonia in whom the above mentioned bacteria resistant to most antibiotics were isolated except for colistin, polymixin B, aminoglycosides, tetracycline and tigecycline. Additionally, caramapenemases were detected, which proves high pathogenicity of this bacteria [19].

\section{Conclusions:}

An increasing number of infections with etiology of R. planticola is observed.The bacterium invades many systems and organs. People with impaired immunity, exhausted, with many coexisting diseases and dialysis patients are exposed to the infection. Therefore, doctors should consider infection with this pathogen in people at risk. The bacterium invades many systems and organs. People with impaired immunity, exhausted, with many coexisting diseases, dialysis patients are exposed to the infection. Therefore, doctors should consider the infection with this pathogen in people at risk. The bacterium invades many systems and organs. People with impaired immunity, exhausted, with many coexisting diseases and dialysis patients are exposed to the infection. Therefore, doctors should consider the infection with this pathogen in people at risk. Avoiding contaminated water, soil and hygiene is prevention. R. planticola infection is characterized by good prognosis. 
The main treatment is to early start antibiotic therapy. Like any other human pathogen, close monitoring of its resistance pattern, especially to carbapenems, is recommended.

\section{List of references:}

[1] Bagley ST. Habitat association of Klebsiella species. Infect Control 1985;6 (February (2)):52-58.

[2] Drancourt M, Bollet C, Carta A, Rousselier P. Phylogenetic analyses of Klebsiella species delineate Klebsiella and Raoultella gen. nov., with description of Raoultella ornitholytica comb. nov., Raoultella terrigena comb. nov. and Raoultella planticola comb. nov. Int J Syst Evol Microbiol 2001;51:925-32.

[3] Xu M, Xie W, Fu Y, Zhou H, Zhou J. Nosocomial pneumonia caused by carbapenemresistant Raoultella planticola: a case report and literature review. Infection 2015;43(April (2)):245-8.

[4] Castanheira M, Deshpande LM, DiPersio JR, Kang J, Weinstein MP, Jones RN. First descriptions of blaKPC in Raoultella spp. (R. planticola and R. ornithinolytica): report from the SENTRY Antimicrobial Surveillance Program. J Clin Microbiol 2009;47(December (12)):4129-30

[5] Yokota K, Gomi H, Miura Y, Sugano K, Morisawa Y. Cholangitis with septic shock caused by Raoultella planticola. J Med Microbiol 2012;61(March (3)):446-9.

[6] Hu AY, Leslie KA, Baskette J, Elsayed S. Raoultella planticola bacteraemia. J Med Microbiol 2012;61(October (10)):1488-9.

[7] Salmaggi C, Ancona F, Olivetti J, Pagliula G, Ramirez GA. Raoultella planticola associated cholangitis and sepsis: a case report and literature review. QJM 2014;107(November (11)):911-3.

[8] Lam PW, Salit IE. Raoultella planticola bacteremia following consumption of seafood. Can J Infect Dis Med Microbiol 2014;25(July (4)):83-4.

[9] William Paul Skelton IVa, Zachary Taylora, Jack Hsub A rare case of Raoultella planticola urinary tract infection in an immunocompromised patient with multiple myeloma Elsevier Volume 8, 2017 Pages 9-11

[10] Ershadi A, Weiss E, Verduzco E, Chia D, Sadigh M. Emerging pathogen: a case and review of Raoultella planticola. Infection 2014;42(December (6)):1043-6.

[11] Kim SW, Kim JE, Hong YA, Ko GJ, Pyo HJ, Kwon YJ. Raoultella planticola peritonitis in a patient on continuous ambulatory peritoneal dialysis. Infection 2015;43(December (6)):771-5.

[12] Olson DS, Asare K, Lyons M, Hofinger DM. A novel case of Raoultella planticola urinary tract infection. Infection 2013;41(February (1)):259-61.

[13] Gangcuangco LM, Saul ZK. A novel case of Raoultella planticola urinary tract infection in a female: comment on 'Nosocomial pneumonia caused by carbapenem-resistant Raoultella planticola: a case report and literature review'. Infection 2015;43(October (5)):621-2.

[14] Cho YJ, Jung EJ, Seong JS, Woo YM, Jeong BJ, Kang YM, et al. A case of pneumonia caused by Raoultella planticola. Tuberc Respir Dis 2016;79 (January (1)):42-5.

[15] Yoon JH, Ahn YH, Chun JI, Park HJ, Park BK. Acute Raoultella planticola cystitis in a child with rhabomyosarcoma of the bladder neck. Pediatr Int 2015;57 (October (5)):985-7 
[16] Hassan Mehmood, MD , Najwa Pervin, MD, Muhammad Israr Ul Haq, MD, Khushbakht Ramsha Kamal, MBBS5, Asghar Marwat, MD, and Muzammil Khan, MD $A$ Rare Case of Raoultella planticola Urinary Tract Infection in a Patient With Immunoglobulin A Nephropathy Journal of Investigative Medicine High Impact Case Reports Volume 6: Pages 1-3, 2018

[17] Serkan Atıc1, Zeynep Alp Ünkar, Sevliya Öcal Demir, Güls, en Akkoc, Nurhayat Yakut, $\mathrm{S}$, erife Yılmazc, Kübra Erdemc, Aslı C, nnar Memis, oglu, Nurver Ülger, Ahmet Soysal, Eren Özek, Mustafa Bakır A rare and emerging pathogen: Raoultella planticola identification based on $16 \mathrm{~S}$ rRNA in an infant Elsevier Journal of Infection and Public Health 11 (2018) Pages 130-132.

[18] Tseng SP, Wang JT, Liang CY, Lee PS, Chen YC, Lu PL. First report of bla(IMP-8) in Raoultella planticola. Antimicrob Agents Chemother. 2014;58:593-5.

[19] Gonzales Zamora JA, Corzo-Pedroza M, Romero Alvarez M, Martinez OV. Carbapenemase-Producing Raoultella Planticola: A Rare Cause of Pneumonia and Bacteremia. Diseases. 2018;6(4):94. Published 2018 Oct 17.

[20] Gian J, Cunha BA. Raoultella planticola chronic bacterial prostatitis with prostatic calcifications: successful treatment with prolonged fosfomycin therapy. Int $\mathrm{J}$ Antimicrob Agents 2016;47(May (5)):414.

[21] Alves MS, Riley LW, Moreira BM. A case of severe pancreatitis complicated by Raoultella planticola infection. J Med Microbiol 2007;56(May (5)):696-8. 